\title{
Gender fluidity as affordance negotiation
}

\section{Mahault Albarracin ${ }^{1,3}$, Pierre Poirier ${ }^{2,4 *}$}

$3 \quad{ }^{1}$ Department of Computer Science, Université du Québec à Montréal, Québec, Canada

$4 \quad{ }^{2}$ Department of philosophy, Université du Québec à Montréal, Québec, Canada

$5{ }^{3}$ Institut de recherche et d'études féministes, Université du Québec à Montréal, Québec, Canada

$6 \quad{ }^{4}$ Institut des sciences cognitives, Université du Québec à Montréal, Québec, Canada

* Correspondence:

9 Corresponding Author

10 poirier.pierre@uqam.ca

11 Keywords: Gender, Gender fluidity, Cultural affordance, Ecological psychology, Enaction, 12 Active Inference, Social scripts.

\section{Abstract}

14 Gender is often viewed as static binary state for people to embody, based on the sex they were assigned at birth. However, cultural studies increasingly understand gender as neither binary nor static, a view supported both in psychology and sociology. On this view, gender is negotiated between individuals, and highly dependent on context. Specifically, individuals are thought to be offered culturally gendered social scripts that allow them and their interlocutors the ability to predict future actions, and to understand the scene being set, establishing roles and expectations. We propose to understand scripts in the framework of enactive-ecological predictivism, which integrates aspects of ecological enactivism, notably the importance of dynamical sensorimotor interaction with an environment conceived as a field of affordances, and predictive processing, which views the brain as a predictive engine that builds its probabilistic models in an effort to reduce prediction error. Under this view, script-based negotiation can be linked to the enactive neuroscience concept of a cultural niche, as a landscape of cultural affordances. Affordances are possibilities for action that constrain what actions are pre-reflectively felt possible based on biological, experiential and cultural multisensorial cues. With the shifting landscapes of cultural affordances brought about by a number of recent social, technological and epistemic developments, the gender scripts offered to individuals are less culturally rigid, which translates in an increase in the variety of affordance fields each individual can negotiate. This entails that any individual has an increased possibility for gender fluidity, as shown by the increasing number of people currently identifying outside the binary.

\section{$32 \quad 1 \quad$ Introduction}

According to a still popular neuroreductionist account of gender, evolution gendered brains to solve the distinct recurrent survival problems faced by ancestral men and women in an environment of evolutionary adaptation (Baron-Cohen, 2003). On such views, gender is often construed as a static binary state people embody based on the sex they were assigned at birth (Geary, 2010; Österman \& Björkqvist, 2018). Cultural studies, however, increasingly understand gender as neither binary nor static (Coney, 2015), a view supported both in psychology (Gabbard \& Wilkinson, 1996) and 
sociology (Linstead \& Brewis, 2004; Valocchi, 2005). On this account, gender is a interpersonal negotiation between individual agents, highly dependent on context; specifically, cultures offer individuals social gendered scripts, which allow them and those they interact with the ability to predict each other's future actions, and understand the interaction scene being set, establishing roles and expectations for everyone involved (Goffman, 1978; Simon \& Gagnon, 1986).

In this paper, we propose to understand cultural social scripts in the framework of predictiveecological enactivism, which integrates aspects of enactivism, notably the importance of dynamical sensorimotor interaction with an environment conceived as a field of affordances, and predictive processing, which views the brain as a predictive engine that builds its probabilistic models in an effort to reduce prediction error. Under this view, script-based interpersonal negotiation can be linked to the enactive neuroscience concept of a cultural niche, as a landscape of cultural affordances (Ramstead et al., 2016). Affordances are possibilities for action whose perception, based both on multisensorial biological (homeostatic, bodily, skilled capacities, etc.) and cultural cues (practices, hermeneutic resources, norms, etc.), constrain which actions are pre-reflectively felt possible. In a modern urban setting, for instance, most people pre-reflectively feel that dark rooms can be lit (a switch somewhere affords lighting), fire-fighters can be called should there be a fire emergency (phones afford calling 911) and social interactions with strangers can be cut-off at will given a few polite niceties (polite conversation affords control over social interactions). Things may go wrong, to be sure, but this is how, ecologically, the modern urban setting is pre-reflectively felt by most. As they act in a given situation, individuals enact a subset of their landscape of affordances as the situation's field of affordance (Bruineberg \& Rietveld, 2014; Kirchhoff \& Kiverstein, 2019). A situation's field of affordance, thus, is the pre-reflexively felt structure of possible actions in that situation. No two agents enact the same field of affordances in any given situation, since fields of affordances depend on multisensorial biological and cultural cues, and no two agents have identical bodies (homeostatically, kinesthetically, etc.) and no two cultures (which can be as finely individuated as the individual) offer identical norms and practices. Since humans share biological and cultural commonalities at various spatial and temporal scales, however, their collective fields of affordances in a given situation thus form a space, in which there is structure at multiple temporal and spatial scales. We call this structure, which will be important to understand the possibility and constraint on gender fluidity, "the fields-of-affordances space". Any action undertaken by a specific human agent in a given situation will bring forth a new field of affordances, belonging to a new fields-of-affordances space, opening new action possibilities, and so on. Scripts, as we will argue, are biologically and culturally constrained sequences of such action-affordance loops, and genders are a subset of those. Specifically, they are the action-affordance loops constrained by the individual's culturally shaped biological body, the way culturally-shaped biological body is perceived by others, the cultural norms that shape bodies, brains and behaviors, and the political forces that shape those norms. This, unlike neuroreductionist views, entails that any individual that enters new situations has a possibility for gender fluidity, that is, of enacting new gendered affordance-actions loops. These possibilities reflect the structure in the fields-of-affordance space: individuality but also commonalities at multiple temporal and spatial scales.

With the shifting cultural norms and embodiment possibilities brought about by a number of contemporary political, social, technological and epistemic developments, the norms of gender are becoming less rigid, which translates into an increase in the variety of scripts agents can negotiate and a concomitant decrease in strength of the normative constraints put on individuals as they move from situation to situation, as evidenced by the increasing number of individuals identifying outside the binary. The affordance negotiation approach allows us a critical stance on the cognitive neuroscience of gender. By integrating the social and cultural backgrounds, power dynamics and

This is a provisional file, not the final typeset article 
embodied reality of the agent, enactive-ecological neuroscience avails itself of the theoretical benefits of standpoint theory and the intersectionality literature. By entering in a dialogue with them, it also opens the possibility of enriching standpoint theory and the intersectionality literature by the finergrained understanding of how cultural norms affect the neural, bodily and skillful action dynamics at play in gender fluidity. The approach thus has the real potential to improve both enactive neuroscience and feminist critical theory, by helping to address the potentially problematic nature of the over-subcategorisation of intersectionality (May, 2014), and the simplistic mechanistic naturalism of traditional cognitive neuroscience, both for theory and for research practices.

\section{Predictive-ecological enactivism}

We claim here that predictive-ecological enactivism is a fruitful framework to understand gender, gender fluidity and gender norms. Predictive-ecological enactivism, as we see it, builds on the enactive tradition in cognitive science initiated by Valera, Thompson and Rosch (1991), the latter being a continuation of many pragmatist and phenomenological traditions in philosophy, on Gibson's (1979) ecological approach to perception, and on the more recent Free Energy Minimization framework developed by Karl Friston (Friston, 2019). This framework will allow us to revisit Gagnon's notion of sexual scripts (Simon and Gagnon 1986), and its conceptualization of gender, leaving us with a notion of gender that has the explanatory power to understand fluidity. In this section, we will briefly introduce each of the components of our framework, explaining how they fit together in a coherent whole.

\subsection{Enactivism}

Cognitive science was born in an attempt to use computational theory, notably the work of Alan Turing (1936), to answer the classical rationalist problem of how humans come to know so much given the poor information they get from the world. Enactivism in cognitive science can be viewed as the classical pragmatist rejection of rationalism (cognition is not in the service of knowledge and truth but of action and survival), supercharged by systems biology and a strong commitment to grounding cognition in life. It is an attempt at understanding cognition not as a representational phenomenon made possible by computation, but as a biological phenomenon, using the formal framework of systems theory (Ashby 1962, Bateson 1972), and especially dynamical systems theory.

Enactivism views cognitive properties on a continuum with biological properties, the properties that give rise and maintain life. Life is understood as the self-organization and homeostatic maintenance of chemical reactions, a combination Maturana and Varela (1991) called autopoiesis. The most basic way for a self-organized autocatalytic set of chemical reactions to achieve homeostasis is to build and maintain a semi-permeable membrane to enclose itself in, making it able to weather punctual changes in its milieu. By doing so, the autocatalytic set of reactions takes its first step in the journey of variational free-energy minimization: it makes viable internal states more probable than states less conducive to self-preservation, hence the viable internal states tend to be revisited more often than less (or non-)viable states. For a living organism, variational free-energy is an information-theoretic quantity that is an upper bound on the surprisal of a set of states sampled from its (internal or external) environment, given a generative model of the causes of the sample. The Free-Energy Principle (Friston et al., 2006) states that any adaptive living organism resists disorganization by minimizing its free-energy. All adaptive living organisms are thus posited to possess a generative model of its environment, at first by its very bodily structure and then by more sophisticated means. By minimizing free-energy, organisms increase the probability that they will find themselves in states 
129 that are homeostatically valuable. Homeostatic value is the most basic form of value, from which all

130 forms of biological values can be explained. This does not mean that humans are limited to valuing

131 things that are homeostatically valuable; to the extent that they do not, they will value things that

132 have no biological value - and that is ok.

133 A more sophisticated way for an enclosed self-organized autocatalytic set of reactions to preserve

134 homeostasis is to be sensitive to conditions inside and outside its membrane, and be able to react

135 appropriately to changes in internal and external conditions (Cisek 2019). At its most basic, this is

136 done by chemical phase transitions, which occur when the presence of some chemical or set of

137 chemicals in a particular (internal and/or external) milieu is sufficient to change the self-organized set

138 of reaction from one regime of chemical interaction to another. Although traditional philosophy,

139 psychology and cognitive science has focused almost exclusively on the relationship between the

140 organism and its environment, no distinction should be made today between internal and external

141 sensing and reacting: internal and external sensing and reacting may use different sensors and

142 effectors but their function is identical: maintaining homeostasis. This new regime may be one that is

143 itself self-sustaining (and thus homeostasis may work to preserve it), or one that, in time, will restore

144 the previous self-preserving regime of interaction. This basic mode of homeostatic regulation may be

145 extended in by sensing and reacting to conditions in their environment, creating dynamical

146 sensorimotor loops of interaction between the organism and its environment. Such sensorimotor

147 loops are observed, for instance, when the sensing of a specific chemical causes each one of a

148 bacterium many flagella to rotate independently, causing the bacterium to tumble, until that chemical

149 is no longer sensed, causing the flagella to unite into a bundle whose rotation propulses the bacterium

150 forward, to another patch where the chemical is present. The passage from homeostasis maintenance

151 by chemical phase transitions to homeostasis maintenance by sensorimotor loop is important for it

152 gives homeostatic value to the environment: some of its states (e.g. presence of a given chemical)

153 afford the organism motor actions that preserve homeostasis, and some don't. Dynamical

154 sensorimotor homeostasis gives meaning to the world (Di Paolo et al., 2018; Thompson, 2007) : 1)

155 sensing and action capabilities are evolved to detect those conditions in the world that are beneficial

156 for maintaining self-organization, and those conditions that are detrimental to it, and take appropriate

157 action; 2) and the organism will be attracted to the conditions that are beneficial and repulsed by

158 those that are not. According to Damasio (Damasio, 2018), homeostasis is the basis for basic affect

159 and feeling: given the proper neurological capacities for building models of their internal and

160 external environment (see below), organisms will experience pleasure to the extent they are in

161 homeostatically preserving states, and displeasure to the extent they are not.

\section{$162 \quad 2.2 \quad$ Ecological psychology}

163 In ecological psychology (Gibson, 1979), objects (situations, states, events, etc.) that afford actions to

164 an organism are called "affordances". Affordances are possibilities for action that the organisms'

165 environment supplies (affords) to those that can perform the afforded action. Organisms are able to

166 perform actions to the extent they possess the necessary body configuration, physiology, and skills.

167 Since birds possess the musculo-skeletal properties to achieve unaided flight (e.g., hollow bones,

168 large muscles to propel the wing, etc), open space (as opposed e.g. to the restricted space of a cage) affords flight to them. Fish possess the proper physiology to extract oxygen from water, and hence water affords respiration to them (as opposed to mammals, even aquatic). Finally, organisms must possess the necessary skills to perform the action, a skill which may be innate (swimming in fish) or learned (flying in birds). Since body configuration, physiology and skills change throughout the organism's lifespan, the affordances it finds in a given environment change as well. Affordances are

174 thus time relative. For a given organism, the set of affordances supplied by its (local, global) 
175 environment at a given time is determined by the content of its (local, global) environment at that 176 time, as well as its body configuration, physiology and skills at that time.

177 The set of affordances an individual (or type of) organism has at a given point in time are called the 178 individual's Umwelt (von Uexküll), or its ecological niche (Gibson, 1979). A more universal 179 (species-wide) notion of Umwelt (or ecological niche) can be given if one considers normal 180 organisms of that species (but then one has to specify how "normalcy" is established), or by 181 considering the set of all possible affordances a species can have (but this make the notion of Umwelt 182 open, for organisms can develop new action capacities — e.g., opening milk bottles for Blue tits—and 183 to transform neutral objects into affordances). Finally, definition-wise, the space of affordances 184 currently available to an organism is called the organism's affordance landscape (Bruineberg \& 185 Rietveld, 2014).

186 We will focus here on an important subset of affordances, at the intersection of enactive and 187 ecological psychology, those we may qualify as homeostasis preserving (or free energy minimizing): objects (situations, events, etc.) in the organism's environment affording actions that allow the organism to maintain itself in homeostatically viable states, that is, minimize free energy. These affordances have value for the organism, and the organism that minimizes free energy will opt for the action afforded by the affordance in its affordance landscape that has the highest value. Following Bruneberg and Rietveld (2014), such affordances can be called solicitations. In the context of enactive-ecological psychology, sensorimotor loops become loops of affordances-afforded actions (which can be called "sensorimotor affordance loops"): given an affordance landscape, the freeenergy-minimizing organism will opt for the action that most minimizes free-energy, which will bring about a new affordance landscape, a new action free-energy-minimizing action, and a new affordance landscape, and so on.

\subsection{Active inference}

Homeostasis, as described so far, is a reactive process (Ashby, 1962), and so are the sensorimotor affordance loops built to maintain it. Sensors tell the organism whether it is within its range of viable states or not, and a controller responds by changing the current (chemical, behavioral) state of the organism. This form of homeostatic control does not require a brain, as the previous example of the bacterium illustrates. Following Cisek (Cisek, 2019), we take it that "the evolutionary history of the nervous system is essentially a history of the continuous extension of such control further and further into the world." Brains allow organisms predictive homeostasis, or what Barrett (Barrett et al., 2016), following Sterling (Sterling, 2012), call allostasis. Instead of passively waiting for internal or external conditions to change, much as an old-time thermostat would do, allostatic organisms try to predict the coming changes in order to respond preemptively to insure homeostasis. One of the most influential recent advancement in neuroscience is the development of a framework, now often referred to as the Active Inference Framework, that understands the brain as a predictive system (Friston et al., 2006). The computational mechanism grounding the Active inference Framework has grown in detail and complexity over the past fifteen years, and we will focus here on the details that are relevant for our purpose. Structurally, the mechanism is a deep hierarchical neural network in which each neuron in any layer is bidirectionally connected to all neurons in adjacent layers below and above it (there are lateral connections within a layer as well). Neurons in the lowermost level are either sensory neurons (of many kinds) or effector neurons, bidirectionally connected to neurons in the layer above, but also transducing signals from the organism's internal and external environment (in the case of sensors) or driving motor or glandular activity (in the case of effectors). Although 
signals flow bidirectionally at all times and in all levels of the architecture, proponents of the Framework find it heuristically convenient to emphasize the downward flow. The computational mechanism (its equations) is set up as a hierarchy of generative models (probability densities) in which each neuron at one level attempts to generate the signal it is about to receive from the neurons in the level right below (that is, a weighted sum of these neuron's activity). For a variety of reasons we won't go into, this is often, but controversially, interpreted as an attempt to "predict" the activity of the neurons in the level below. Once a neuron in a layer has generated the signal it is about to receive (the "prediction"), it compares the generated signal to the signal it actually receives from the layer below, and then sends the difference, termed "prediction error" in the standard interpretation, to neurons in the layer above.

Each layer encodes a set of generative models in a superposed fashion, each of which could be used at a point in time to generate the upcoming signal. On a short timescale, the upward flow of signals ("prediction errors") serves model selection, that is, selecting which generative model will be active in a given layer. On a longer timescale, the upward flow of signals serves learning, that is, changing the connection weights between layers and thus changing the models encoded in a layer. This allows the brain to minimize the prediction error it generates, which, under certain assumptions, minimizes its free energy. The brain can also minimize its prediction error, and thus its free energy, by changing the organism's internal and external environment by the action of the organism's effectors, a process called "active inference". If the environment is viewed as a model of the organism, then active inference can also be understood as model selection (Constant et al., 2019). The environment can also be changed at different timescales. At the shortest of timescales, any movement from the organism changes what is sampled from the environment, without the environment changing itself; at increasingly longer scales, objects can be moved, structures can be built, dynamics set in motion, institutions established, cultures evolved.

The hierarchical nature of the architecture imposes an order to the system's signal generating activity. Neurons at the sensory periphery, from cones and rods to inner hair cells, and from to nociceptors and baroreceptors to muscle spindles, receive very local and short timescale signals and this is what they will attempt to generate; similarly for motor neurons: they generate the local and short timescale signals that activate muscle fibers. Neurons at next level up will receive the prediction error signal from the layer below. Initially, when the layer below has no working generative model, the error is large, and that second layer gets to learn a model of the environment as filtered by the first layer. As the first layer improves at generating incoming signals, it sends less and less signal upward, except in novel environments and situations. The results of this arrangement is that higher and higher layers encode regularities at larger and larger spatial scales, longer and longer timescales; regularities that are more and more abstract with respect to the raw sensory signals.

The move from low level to high level in the hierarchy also sees a progressive integration of signal from multiple sensory (interoceptive, proprioceptive and exteroceptive) and effector (motor, endocrine). As signals from multiple sensory and effector hierarchies are integrated at generative models embody the affordances of objects and situations: neurons attempt to generate the signal which, given context signals from levels above and prediction error signals from below, would (predictively) best maintain homeostasis if it were sent down the hierarchy (and will receive an interoceptive feedback signal that contains information about actual maintenance of homeostasis). Generative models at higher levels thus encode the probability distribution of affordances. And because of the hierarchical nature of the generative models, this means these probability distributions of affordances are arranged in a nested hierarchy: restaurants afford many actions (eating, spending money, sitting, meeting people, going to the restroom, etc.) that themselves afford many actions. In 
265 the case of humans, many of these affordances are cultural: actions afforded by a culturally designed objects (e.g., a light switch), practices (e.g., the multiplication algorithm) or institutions (e.g., education): the light switch affords lighting dark rooms, the multiplication algorithm affords multiplying any two numbers, and education affords, e.g., working in certain fields. Affordances are cultural when it is human culture that designed its objects, practices and situations and sustains the possibilities for action. It is human culture that designed the situation in which a switch on a wall affords illuminating the room when it is dark and sustains the switch's affordance (by enabling the processes that keep electricity coming to the switch and bulb, ensure the manufacture of bulbs, etc.). The existence of cultural affordance opens the possibility for the presence of sensorimotor culturalaffordance loops: sensorimotor affordance loops where it is culture that sets up and sustains the artifacts processes such that some objects or situations afford actions.

\section{Cultural scripts in ecological-enactivist framework}

\section{From sensorimotor affordance loops to cultural scripts}

A script is a form of knowledge structure about the sequence of events to be expected in a given setting. A typical example is the restaurant script (Schank \& Abelson, 2013). Upon entering a (traditional, non fast-food type) restaurant, one expects to be seated, met by a person assigned to wait on them, offered a menu, and so on (anyone familiar with the restaurant script can complete the sequence and is aware that many details have already been left implicit). This knowledge is thought to be encoded in a memory structure that evolves over time as individuals are exposed to multiple varied instances of the setting (e.g., multiple trips to restaurants). An important point to note about scripts is that they pair normative sequences of perceptions and actions. Upon opening the door, most restaurants will offer customers a familiar perception (multiple tables and chairs, dining customers, waiting staff), perception of which should prompt one to stop and wait to be met by a member of the waiting staff (or someone specifically hired to seat customers). To the extent an individual follows the norms of a particular script, he or she can be said to play a role in that script. In the above example, the individual, by behaving in a manner that respects specific norms of the restaurant script, plays the "customer role".

The typical example, from the cognitive science literature, is an example of a cultural script, because it is embedded in a culture that created the objects and situations that make up the script (which can be seen as the script's props) and prescribes (and sometimes enforces) the norms individuals must follow to play a role in the script. This does not mean that all scripts are cultural. Natural scripts are set up by the organism's need to maintain its precarious self-organization in its natural environment. Although these may be quite basic (e.g., bacteria chemical gradient ascent or descent scripts), they may also be quite complex (e.g., the prey-predator scripts). Though the roles in such scripts may be learned, following their norms is rarely a matter of choice. Although natural scripts are interesting in their own right, and we should never underestimate their presence in human cognition, we shall focus here on cultural scripts. We shall argue that cultural scripts are better understood within the predictive-ecological enactive framework.

303 Recall that affordances are possibilities for action offered by objects and situations, which constrain what actions are pre-reflectively felt possible. We saw that an important enactive-ecological psychology concept is the sensorimotor cultural-affordance loop. From the enactive-ecological 
307 follows, we shall explain how to view scripts as such loops and show the explanatory power thereby

308 gained by the concept of cultural script.

309 Let's go back to the restaurant script. Cognitive science traditionally construes scripts as internally

310 memorized sequences of actions, which is a representationally and computationally costly, and

311 sometimes brittle, way of structuring behavior. Enactive-ecological cognitive science offloads some

312

313

314

315

316

317

318

319

320

321

322

323

324

325

326

327

328

329

330

331

332

333

334

335

336 of those representations and computations to the environment. One does not learn a script but becomes sensitive to the affordances it presents and skillful at producing its afforded actions. On the active inference view adopted here, one becomes sensitive to an affordance when one's (hierarchy of) generative models predict the affordance and prepares her whole organism to act upon the possibility offered by the object or situation. At that point, the body's (including the brain's) activity gives meaning to the object or situation (Johnson, 2007). It is, expanding on Barrett (Barrett et al., 2016), the organism's current interpretation, its current concept, of the object or situation. Since similar agents, or the same agent in different situations, may generate similar interpretations, concepts on this view are polysemic. Acting upon the possibility offered normally brings a response from the environment (either because of its structures or mechanisms or because the action changes the affordances of other humans who will normally respond in a culturally normed fashion), which brings new affordances, and thus new actions. One enters a restaurant in part at least to eat and thus ensure the short-term maintenance of variables within their homeostatic range, thus minimizing free energy. Upon entering the restaurant, one enters into a culturally constructed environment that affords eating, but is first met however with a setting whose norms culturally afford waiting. Violating this norm might bring a response from the restaurant's staff or owner that affords existing the restaurant. Waiting, on the other hand, will present staff with a situation that affords offering a seat at a free and clean table as well as a menu to anyone that waits (obviously, this could be described in much more details). Being seated at a clean table with a menu affords consulting the menu to choose an item, and so on. One progressively masters the script as her brain's higher-level generative models predict larger spatial and temporal events associated with the script and, if necessary, as her body's physiology adapts (bones thicken, neuromuscular connections grow, etc.) the actions offered by the script's affordance. At that point, one possesses a multilevel, polysemic concept of the cultural script, some aspect of which are social (those that concern interactions with other humans).

A social script can be thus seen as a meaningful script an individual has constructed from living in society, and having to exploit its group dynamics. Specifically, groups afford different possibilities to people who perform or conform better to their norms. Resources are also offered to people who conform to the group norms. An example of this can be found in urban cultures through informal giving practices. An individual is never forced to acquiesce to a request from a member of the group, but refusing such a request may mean that one is then cut off from the community should they need help in turn (Hornsey et al., 2003); (Griskevicius et al., 2006). Access to resources is thus conditional on the capacity of the individual to conform to group norms (or habitus, Bourdieu (1990a, 1990b)), but is not sufficient. Certain group dynamics afford different things to different statuses of their members, regardless of their conformity to group norm. Certain groups thus predefine a member status which grants more power, and thus more affordances over resources than others. These cultural affordances can be defined as deontic affordances, since they do not define what an individual can do, but what an individual should do.

\section{$350 \quad 3.2 \quad$ Cultural scripts are fluid}


We mentioned that traditional, internalist and representational, cognitive science construes scripts as internally memorized sequences of actions. Apart from being representationally and computationally costly, this way of structuring action makes cultural scripts rigid and difficult to change. On this view, the introduction of fast food restaurants in the fifties and sixties would have required learning a new sequence of action. Traditional cognitive science presumes that representations can be added at little computational cost (like when one writes information into a computer's random-access memory. But a cognitive science more cognizant of neuroscience understands that learning new representations is a long process. Learning the new sequence of actions associated with fast-food restaurants would have required many trips to fast-food restaurants and the frustration of many suboptimal experiences in such restaurants (and thus probably doomed the enterprise from the beginning). On the predictive ecological-ecological view, the introduction of fast-food restaurants itself introduced the new sequence of actions. Prior to the introduction of fast-food restaurants, the inside appearance of restaurants perhaps provided some information about the type and quality of food to be found in the restaurant, but only afforded the waiting action upon entering. Fast-food restaurants introduced a new affordance, supplied, e.g.,, by the gaudy inside appearance of fast-food restaurants. This particular appearance affords walking up to a service counter upon entering the restaurant. All individuals had to do is become sensitive to that new affordance. In time, with cultural evolution, the inside appearance of restaurants became clearly distinguished: posh affords waiting to be seated, gaudy affords walking to the service counter. On this view, the fact that most fast-food restaurants have a distinctive appearance is not incidental, but a very part of the cultural script.

The rest of the fast-food restaurant script is made-up of affordance-action pairs the individuals already had mastered, either as part of other scripts (from shopping at stores generally, eating in school cafeterias, etc.) or as part of the previous restaurant script. On the predictive enactiveecological account, cultural scripts are loosely coupled cognitive structures that can be decoupled from their original script and recoupled into other scripts as the environment demands. This is a feature we will call their porosity. This can be done because mastery of sensorimotor affordance loops sits at a lower level hierarchical level that learns faster than the higher levels where the full temporal and spatial dimensions of the script are integrated. As a result, cultural scripts are much more fluid and quickly responsive to environmental change than traditional scripts. And as we saw with the introduction of fast-food restaurants, it also makes it much easier to introduce now cultural scripts, thus promoting cultural creativity. We now see how all of this can be used to understand gender and fluidity.

\section{The fluidity of the gender script}

To illustrate the fluidity of cultural scripts, we can use the gender script as an example. Most cultures produce and maintain a gender script, and it has large scale ramifications on the order of social gendered norms and prescriptions. Social practices promote certain bodies over others in relation to gender ideals, and any derogation from the norms is punished by the group. While the gender script seems stable over time, it has known some notable changes both in the recent and distant past that were well documented through narrative and iconic records. The most recent changes have been more drastic however, as gender has taken on a less binary nature, reflected in the myriad new identifications. In this section, we identify gender as a cultural script, and show how the structure of cultural scripts allowed gender to be fundamentally fluid, until the script itself changed.

\subsection{Gender is a script}


395

396

397

398

399

400

401

402

403

404

405

406

407

408

409

410

411

412

413

414

415

416

417

418

419

420

421

422

423

424

425

426

427

428

429

430

431

432

433

434

435

436

437

438

439

440

441

Gender is a set of practices that segregates human affordances (possibilities for action) according to biological (i.e., GGG - which is mostly binary) sex. It sets up distinctive affordance landscapes for human males and females. Specifically, by contrasting masculine to feminine, those categories have their own segregated associations. Actions associated with either of those conceptual clusters will be afforded to agents embodying either of the gendered poles, and refused to the others. In this sense, gender is a set of affordance-action associations with ramifications into practice. Understood this way, it is possible to refer to Butler's notion of performativity (Butler, 2009). Gender is meant to communicate a social identity through identifiable actions. Butler does not view performativity as conscious, but it entails that agents will enact what they understand to convey an identity they have internalized. Social scripts follow this exact pattern of pre-reflective rule following according to a social position. Butler also proposes that an individual's performance has some possibility for reappropriation, and that agents have some agency in the way that their performance is expressed and socially understood. We can link this to the previously mentioned polysemy of scripts, and their capacity for change. Scripts do not change simply by virtue of existing, but because of the actions of agents and their interaction with the world. Finally, Butler proposes that individuals cannot simply escape social norms, because they need to remain intelligible. Even counter-discourse is somewhat subjected to the main discourse, as it directly hinges on this intelligible common discourse, and hence must define itself through opposition. This is directly relatable to scripts in the sense that scripts fashion our perception of the world, and our understanding of social spaces. Scripts surround conceptual spaces and render the world intelligible. In this sense, a gendered world is a world where interactions between gendered agents are predictable and testify to social intentions. By following these associations, agents also perceive the world through their own possibilities, based on their internalized positions. These affordances shape their effectual understanding of the world, and others around them.

The performativity of gender is thus understood as the set of norm-regulated actions individuals undertake given the culturally determined pre-reflective cultural-affordance landscape generated by the action they undertook. This performativity is maintained by the cultural frame around the individual. Indeed, agents do not simply wake up with a choice. They are pushed and pulled in those directions by institutions and social representations. Agents are constantly reminded of the framework within which they operate, and their affordances are made salient based on the cultural codes at play. Culture provides a segregated affordance landscape to males assigned at birth and females assigned at birth and gendered individuals perform the actions solicited by their normative affordance landscape (e.g., a picture on a door affords peeing to one gendered individual but not another). More than simply proposing categorical separations, like physical segregation, affordances are linked separately in terms of sequences. For instance, the bathrooms are laid out differently, and allow different affordances for men and women. Similarly, toys for boys and girls propose different affordances. Toys for boys usually afford motor activity and competition between boys. In contrast toys for girls usually afford quiet caring and cooperation between girls. There is thus an intrinsic set of afforded actions culturally encoded in the relative toys.

These segregations are not entirely arbitrary. They have some basis in a sense of complementarity. This structure upholds the heteronormative framework. In that frame, the sense of complementarity between men and women justifies that they need each other, and are naturally drawn to each other. Thus these genders are defined in relation to one another, as a sort of complementary all encompassing whole. Feminine is defined against the masculine, with a different social valence associated to it. That is, masculinity is associated with more desirable qualities overall, albeit not desirable for any given individual to possess. This also serves to uphold the social hierarchy that places men above women. By reinforcing these narratives, affordances are offered to men in a way

This is a provisional file, not the final typeset article 
442 that they are refused to women. Scripts panning over lifetimes are drawn in this way with a clear 443 sequence (Jordan-Young and Karkazis 2019).

444 Women, for a long time, were associated with care, and home making, which justified that they were not supposed to have a job, or get away from their children for too long (Shome, 2011). The cultural life script of a woman was filled with affordances meant for her to grow up under the parent's supervision, keep her sexuality to herself until she found a man to marry, have children, care for the children until they left, whilst taking care of the home, and finally, redirect her attention to the husband when the children were gone, or eventually her children's children. A woman's life was constrained by affordance landscapes centered around service and compliance. On the opposite, a man's cultural life script was filled with affordances offering sexual exploration, a career unhindered by either the choice to marry and have children or not, and self-affirmation (Richardson, 2010).

As we saw, cultural scripts also define the roles individuals have to perform, socially or for themselves, and the interpersonal scripts to be enacted amongst one another. Specifically, cis-heteronormative cultures would have males interact with females in a dominant manner, and females in a submissive manner (Buss, 1990; Herron et al., 1983). Specifically, beyond these larger scripts about life sequences, smaller weak scripts also define smaller scale sequences. For instance, women and sexuality are associated in contradictory manners. Woman can mean sexuality in a negative light since it is reminiscent of prostitution. Women whose actions afford sexuality are considered socially unacceptable since they threaten the social order of patrimony and legacy (Conrad, 2006). Hence, sexual unavailability is considered preferable. However, women are also objectified and portrayed as sexual objects of desire for men. Desirable women are preferable to sexually undesirable women, because the latter do not suit the tastes of men. This places women in the ambiguous position of only being able to receive sexual attention, but not to offer it, passively affording sexuality but denied actions that afford it (Conrad, 2006). This is a passive position. In contrast, men are considered better prototypes of their category if they can engage successfully in sexual conquest. This is an active position (Buss, 1990). This dichotomy entails a sort of natural sequencing where performing masculinity entails initiating and pursuing, whereas performing femininity entails receiving and resisting. In a typical seduction scenario, it has some ramifications about the sequence of events that may unfold. A man will possibly attempt to engage a relationship, and a female will try to elude this relationship for however long it is still acceptable for her to do so without losing the man's desire. The asymmetric valence of the categories men and women belong to, and their complementarity has some direct impact on the logical sequencing between the categories that become deployed into temporarily nested social scripts. These positions, based on categories to enact and associations to avoid, as well as their sequencing create identifiable roles for agents to embody. Roles simply define clusters of scripts that an individual will embody to guide their behavior in different settings in relation to socially sanctioned goals (Laner \& Ventrone, 2000). A role acknowledges that individuals are positioned in the world, and do not simply perceive all possible affordances equally. Certain affordances, based on the script clusters the individual embodies, will be made more salient, and allow for normative social scenes to unfold predictably and relatively smoothly. Examples of such gendered roles include the mother for women, and the breadwinner for men. In more contextual situations, the submissive role expected of women can take many forms and will unfold in relation to the dominant masculine role, or the elements relevant to the situation. scripts of that role according to social expectations, they are punished or ostracized by the group (Krane, 2018). Derogating from a role is risking to be socially unintelligible, and thus, unpredictable, 
affording "the wrong" actions gender wise. This lack of predictability can be bothersome for other agents for a host of reasons, including the generation of prediction errors, the breakdown of cultural sensorimotor loops, and the social threat to hierarchy and functioning (Albarracin et al., 2020; Krane, 2018). Certain individuals or groups have more to gain from the social roles being maintained. Those whose valence is high will probably benefit more from a given system being upheld, and may be more active in trying to maintain this social order. It is even possible that people who highly benefit from that system may not even perceive the issues it presents for other agents because their perceptual field is made of their own set of affordances, defined by the scripts they embody.

\section{5}

\subsection{Sources of fluidity in gender}

We explained above that scripts guide behaviors both in terms of pre-reflective affordance-action loops that are embodied by an agent, and in terms of ecologically constraining nested structures (e.g., sequences) of affordances. However, this does not mean that scripts are entirely fixed, and devoid of the possibility for interpretation and negotiation between agents. Suggesting so would be denying the cognitive reality of human understanding and perception. To begin with, we have identified that agents have different affordance fields constrained by their position in society. This very first point of anchor shows how two agents in the same situation, sharing a set of socially complementary scripts may not see the situation the same way. even to the same people in different scenarios. Two individuals with similar scripts may choose to perform it differently. This performance still needs to serve as an intelligible tool of social prediction and communication, and individuals negotiate their gender in relation to a sort of give and take. Individuals in a situation wish to express a role they embody, and this role will be partly defined by what affordances for the completion of this role other agents propose. For instance, if femininity is defined as softness and care, and an agent finds it appropriate to embody this role but the interlocutor's version of gender is to refuse such expressions, expressing femininity may be difficult in those terms without causing interpersonal, or even social unrest. Hence, the same agent may wish to express a different part of femininity, or express it slightly less so as to be less problematic to the interlocutor. Similarly, if the masculine script is to display successful social conquest, and the agent wishes to embody this role, but the social setting is only made of other agents who value other parts of masculinity, it might be less beneficial for the agent to display these conquests, and they may turn to another part of the script. The contextual expression of a script thus depends on the agents present and their respective understanding of those scripts. Social dynamics emerge thus, in a semipredictable manner, when accounting for individual variability.

However, culture is an overarching structure, and a hierarchy still puts one format of femininity and masculinity at the top, referred to as hegemonic by the literature on gender (Connell, 2014). This is achieved through massively acquiesced (or imposed) narratives about ideals. Iconic and literary representations offer a stable and coherent picture of what constitutes the gendered ideal. This ideal is upheld by institutions and groups who stand the most to gain from it. For instance, for the longest time, the married woman was the gender ideal (Pompper, 2016). And it was thus impossible for unmarried women to have a job, or open bank accounts. Similarly, motherhood was part of hegemonic femininity, and careers in the sectors of care still consist heavily of women, while the environment for women in STEM fields, typically masculine professions, is notoriously toxic (Cheryan, 2012; Dwyer, 2013; Hazelkorn, 2018; Mangan, 2012). Women are pushed out of the fields in droves, even when there are governmental efforts to push girls into STEM fields educational paths. 
532 Thus, gender is not only defined against its binary opposite, but also against itself. Femininity is not a

533 unitary field of one prototype, and neither is masculinity. Feminine and masculine representations

534 and interpretations measure against the hegemonic prototype, and cluster around different types of

535

536

537 enactment (Allan, 2009; Paechter, 2003). This creates different prototypes in turn, all hierarchically positioned. Different dynamics are made possible by this hierarchical variety, and the interactions between the two sides of the binary gender frames. This entails that each individual negotiates their social position in relation to other members around them and gets to renegotiate this position when different versions of the binary poles are presented.

Gender expression (or action from the action-affordance pair, or butlerian performance) is also renegotiated when other scripts come into play. For instance, it is not always advantageous to be a woman in a male-dominated industry depending on personal goals, and ranking in the industry (Corcoran-Nantes \& Roberts, 1995; Wright, 2016). The same is not true in an equal gender distributed industry, or a female dominated industry (Gardiner \& Tiggemann, 1999). Thus, the advantageous behaviors are mediated by the gender of the individual, and the perception of that gender in relation to its coherence with the chosen role by the other members of the group. Thus, if class scripts and gender scripts intersect, their manifestation may be different. It may also be different when meshed with the manifold possible combinations of other social agents. Intersections of race may also change the way gender is defined and expressed, or even valued depending on the community where the agent is embedded (Gardiner \& Tiggemann, 1999; Murphy et al., 2013; Staples, 2005). African American femininity expression may be differently valued in black communities as opposed to white dominant society. A blatant example of this is hairstyles. Typically, African American hairstyles may be valued in BIPOC community, and allow creativity to manifest, while they will be considered unprofessional by the white community, and hinder access to employment and promotions (Powell, 2018). This dual valence entails that a given individual will have to adapt their expression to the type of valence they are faced with, usually in relation to the homogeneity of parts of the intersecting identities. This entails a natural polysemy of gendered script, as well as a variety within those gendered scripts. Agents who can embody this variety have within their concepts a large array of clusters they can draw from at different times.

560

561

The previous examples also highlight the contextual reality of gender. Any given individual may be more advantaged from embodying one version of a script over another at any given time. Agents with a larger variety of available scripts have a greater capacity for adaptation to most situations. It is thus highly adaptive for agents to have a greater capacity for change in their repertoire and be less constrained while staying intelligible.

\section{$565 \quad 4.3$ Gender has changed and expanded}

566

567

568

569

570

571

572

573

574
As previously explained, scripts have a form of porosity as well, due to their loosely coupled structure. The fluidity intrinsic to scripts is due to their polysemy, but agents integrating the scripts do not acquire them once, and forever hold them as they are. Agents are capable of integrating new affordance and new associations. Clusters of associations can arise in social settings due to stable intersections for instance. The condition for a change to be made cultural is that it can be picked up by other agents, thereby gaining social communicability. Hence, when an agent is capable of associating a set of behaviors to a concept they already have, their associations clusters expand. If we come back to the examples of the different manifestations of gender in terms of hierarchy, one agent is still capable of recognizing the gender expressed by another agent, even if it is not fully compliant 
with the social prototypes. Moreover, communities can form around specific manifestations of gender expression, and create a valence around this manifestation.

577 If in the general society system, new categories become more valent, different expressions of gender may become the new hegemony. For instance, the married housewife no longer represents the general social ideal. Issues of class and ideology have shifted the valence from caring mother to career superwoman. Different sub communities with different social attributes still value different types of femininity (Paechter, 2003, 2006; Schippers, 2007; Valocchi, 2005). More urban settings will value career women over mothers, while very religious communities will reverse that valence (Edgell \& Docka, 2007; Istenič, 2007; Jain et al., 2018). Sub-communities can easily form and gather in urban settings because the density is higher and the probability of similar people finding each other follows. Hence, urban settings are constituted of pockets of internal different valence that individuals may have to navigate over time (Nicholls, 2008). Hence, they are exposed to a variety of versions of scripts differently valued and must themselves adapt their own expression to each setting by integrating these versions to their concepts of the script in order to stay personally coherent. This promotes expansion of the script clusters for individuals exposed to this variety. For any given individual, associations to gender roles become larger. For instance, available behaviors for women went from being entirely segregated from masculine behaviors to overlapping strongly with most aspects of masculinity. There are still some elements of masculinity that are frowned upon for women in most cases, like the presence of face-hair. Hence the segregation of this aspect of scripts is still relatively defined.

Similarly, technology allows people with similar conceptual clusters to find one another without having to be physically close (Code \& Zaparyniuk, 2010). Communities can form and develop enough conceptual density that their script cluster develops an internal valence. The possibility for people with alternative models of sexuality can find each other this way. A good example of this is BDSM and LGBTQA communities developing online, and creating their own codes to communicate, as well as normalizing role models. These role models also create intelligible performances for members of the groups to be able to find one another and communicate. In the same way, versions of

602

603

604

605

606 gender can gain some community traction, and turn into larger and more intelligible scripts by virtue of being adopted by multiple people to communicate with one another. Because individuals are exposed to a variety of sub-communities, and must negotiate gender in contact with them, our concepts of gender grow just as though they were physical communities. Famously, communities promoting a very specific version of masculinity have grown online in a way that has become recognisable from outside the community. Incels, for instance, are very specific about the expression of their masculinity, and the way they wish to negotiate it with women (Scaptura \& Boyle, 2020). Alternatively, furry communities have developed alternative codes for gender that intersect with specific iconic codes and are also intelligible from outside the community (Satinsky \& Green, 2016).

All these variations have vastly contributed to the expansion of the masculine and feminine gender clusters of scripts to the point where they are not clearly separate anymore. Under the larger concept of masculinity can be found many scripts also suitable for the larger script of femininity. In this way, the concepts are not segregated anymore and the associations between the categories have expanded to largely overlap, they may not allow for easy segregation of the gendered patterns and ultimately for predictability. The gendered script may have reached a point of assimilation where it may not be possible to continue assimilating concepts usefully. Specifically, gender may have reached a point where it will either be entirely deconstructed or reorganised.

\subsection{New possibilities due to conceptual fracture}


620 This new overlap and expansion have given rise to a new accommodation of the gender scripts. New

621

622

623

624

625

626

627

628

629

630

631

632

633

634

635

636

637

638

639

640

641

642

643

644

645

646

647

648

649

650

651

652

653

654

655

656

\section{7}

658

659

660

661

662

663 clusters and categories have developed into new possible identities. Similarly, the porosity of the gendered concept has allowed agents to see them not as segregated and stable, but as fluid and possibly interchangeable. Some individuals have become more likely to accept that their gender identity does not have to directly match with their sex assigned at birth (Deutsch, 2016). This suggests that the categories around gender and naturality have distanced due to the loosening of the gendered segregations. In the same way, new possibilities for identifications that mix both masculine and feminine components have become possible and normalized. Bi-gender, androgyne or genderfluid individuals may change their expression from day to day, or consistently mix culturally clear elements of the binary script (BrckaLorenz et al., 2017). Alternatively, individuals have entirely reconstructed genders outside of the bounds of this initial binarity. People identifying as non-binary, aliagender, aporagender or agender have entirely come out of this conceptual boundary (McCarthy et al., 2020).

While not entirely mainstream yet, these identifications are being adopted increasingly, mostly with the younger population, who by definition has been less exposed to more traditional gender roles (Richards et al., 2016). These gender identifications allow new script clusters and affordances for the people identifying within them, which is also identified as one of the reasons to adopt them. Feeling like one's affordances represent how we feel, specifically, feeling like we can behave and present in a way that feels appropriate to ourselves is a specifically salient reason to choose a specific identity label (Weichold \& Thonhauser, 2020). Choosing the label signals to other members of our community how we wish to be read, it normalizes the cluster we embody, and it allows individuals to reinforce their affordance field. An individual thus predicts themselves, through this selfidentification in a certain set of states, and minimizes the errors or this prediction by acting in order to tend towards those states. It may become problematic when the self-identification is not reflected by the social group, maintaining a certain degree of prediction error.

Indeed, outside of the communities who can easily read these clusters, people adopting these identifications may thus still face some resistance. Which means their affordance fields are complex interactions of their very own intersections and the way in which the environment will react and constrain them. Embodying their intersection may also prove troublesome in mainstream society since their bodies will be read with difficulty, or brought back into the binary folds, as nonprototypical expressions, and therefore lower on the social hierarchy. As individuals continue to embody these identifications, they will slowly influence gender expression possibilities, and their communities may grow to points where they become intelligible by an increasingly large portion of society. They will thus impact their niche, and push the conceptual clusters to continue evolving, and may in time create a system that allows for more options than binarity. As we move out of binarity, it is also possible that the possibilities for embodiment will change, and our entailed concepts of sex and sexuality may evolve as well.

\section{Conclusion}

By integrating the social and cultural backgrounds, power dynamics and embodied reality of the agent, enactive-ecological neuroscience can benefit from the cultural nuance and complexity contained in the intersectional frameworks. Intersectionality, largely developed by black feminists (Crenshaw, 2019), allows us to conceptualize the different positions an individual can experience that cannot be abstracted from their power relations and social context. Specific difficulties arise when different axes of power meet and shape social constraints. Similarly, cultural narratives shape 
664 categories and performativity for agents (Christopher, 2013). When narratives overlap they may be 665 contradictory, or compound into emergent properties, or need to be flexible in order to allow social 666 navigation between social settings that make salient different parts of one's identity. A good example 667 of this phenomenon is exemplified in linguistics with Code Switching. Through Code Switching, 668 agents rely on the flexibility of their identity to adapt to their context by changing linguistic tools as the affordance allows (Genesee, 1982). This phenomenon has neurological implications. Without taking into consideration the neurological impact of the variety of social identifications, it would not be possible to identify this phenomenon. Similarly, understanding the complexities of the perceptual field for individuals is usually derived from universalised models. While these models acknowledge the specific impact of linguistic skills, they do not necessarily take into account the social position of the individual in relation to their interlocutor (Moreno et al., 2002).

In a very similar manner, gendered differences in neurological studies are assumed in a fundamentally binary manner and assumed to be the source of differences (Kim et al., 2014). But the fluidity of gender entails that a moment to moment approach to neurological differences, as well as a relative social position to an interlocutor or a social context may have important effects on the results (Shields, 2008). By highlighting the role of social context on cognitive reality, we also highlight how these differences may not be intrinsic but socially caused and related to specific dynamics in the outside world. A universalist position would thus not be suitable because social contexts are subject to change, and more complex than can be reduced to rigid binary modalities (Mcfarland \& Pals, 2005). This also highlights the very real possibility that changes can be enacted by shifting the environment in a meaningful way around individuals, with impacts on deep cognitive levels. This conception is directly at odds with positivist interpretations of data. Specifically, it entails that some types of data are potentially more meaningful than they were considered before (Wainwright, 1997). Testimonies for instance, while qualitative, carry more power because they are anchored, as opposed to the assumption that bias is something that science must get rid of. Neuroscience may benefit from integrating experienced narratives in order to study specific intersections, and the way that contextuality influences cognitive processes. Some authors, such as Choudury and Pitts-Taylor have already begun such endeavors by bringing closer material feminism and neuroscience (Choudhury and Slaby 2011; Pitts-Taylor 2013).

The very concept of discrete categories (binary genders) may be challenged by intersectionality. The problem lies in the necessities to qualify specific intersections (Shields, 2008). Integrating cultural narratives into our measurement of differences and commonalities may help identify a more finegrained analysis of phenomena. For instance, being a "woman", which is a large scale identity label, may feel less meaningful than being "African American" in the day to day life of an individual (Sullivan and Esmail 2012; Thompson 2001). Her identity may be more complex, and related to the exact ethnicity she identifies with, the setting in which she grew up, the social narrative around this ethnicity in relation to whiteness and other ethnicities and the fluidity of the identities. The lived reality of these intersections of power can have a real neurological impact, and it is possible to formalize them through a dynamic account of these interactions by starting from the direct individual narrative recounting and the meaning they assign to it.

On the other hand, intersectionality in feminist research knows its own challenges. The very strength mentioned above also renders the results given by our current research methods difficult to interpret (Shields, 2008; Valentine, 2007). Research usually tends towards generalization, and the very center of intersectionality and feminist research is the place the individual reality back to the center-stage. Similarly, interactions of power and identity do not only influence members of oppressed groups. There is an asymmetry in the way identities impact cognitive functions based on the group's place in 
710 a social hierarchy. Hence, without a mechanistic account, it can become difficult to relate relative

711 views on different social phenomena and agents (Flintoff et al., 2008; Shields, 2008). Finally,

712 intersectional feminist research often has a qualitative nature, which is considered to carry less

713 epistemic rigor than quantitative studies (Delmar, 1970; Lawson, 1995; Randall \& Phoenix, 2009;

714 Reinharz et al., 1992). By offering dynamical accounts, it is possible to find the common ground for

715 generalization that does not erase lived reality, and draw upon categories that do not represent the

716 fluidity of the social self. By connecting neurocognitive models to standpoint theory and the

717 intersectionality literature, we can thus offer a more fine-grained understanding of how cultural

718 norms affect the neural, bodily and skillful action dynamics at play in gender fluidity.

\section{Manuscript Formatting}

\section{$720 \quad 6.1 \quad$ Headings}

721 You may insert up to 5 heading levels into your manuscript as can be seen in "Styles" tab of this

722 template. These formatting styles are meant as a guide, as long as the heading levels are clear,

723 Frontiers style will be applied during typesetting.

\section{$\begin{array}{lll}724 & 7 & \text { Conflict of Interest }\end{array}$}

725

726

727

728

729

730

\section{1}

732

733

734

735

736

737

738

739

740

741

742

743

The authors declare that the research was conducted in the absence of any commercial or financial relationships that could be construed as a potential conflict of interest.

\section{Author Contributions}

M.A was the lead author and devised the links between gender theory and enactive theory. P.P was the second author and worked more closely on the cognitive science aspects (enactivism, ecological psychology and active inference), laying out the foundations of theory used in the article.

\section{$9 \quad$ Acknowledgments}

We wish to thank Mylène Legault for her help with this article. We also wish to thank Maxwell Ramstead for discussions that helped us move forward with concepts of active inference. P.P. wishes to acknowledge funding from SSHRC grant 435-2020-0846.

\section{References}

Albarracin, Mahault, Axel Constant, Karl Friston, and Maxwell Ramstead. 2020. "A Variational Approach to Scripts." https://psyarxiv.com/67zy4/download.

Allan, Alexandra. 2009. "Masculinities and Femininities through the Years: Boys and Girls Constructing Gender Identities through Communities of Practice." Sex Roles. https://doi.org/10.1007/s11199-008-9533-0.

Ashby, W. Ross. 1962. "The Set Theory of Mechanism and Homeostasis.” Mechanisms of Intelligence: Ross Ashby’s Writings on Cybernetics. Intersystems, Seaside, Pp--49.

Baron-Cohen, Simon. 2003. "Men, Women and the Extreme Male Brain.” London: Penguin. 
744 Barrett, Lisa Feldman, Karen S. Quigley, and Paul Hamilton. 2016. “An Active Inference Theory of Allostasis and Interoception in Depression." Philosophical Transactions of the Royal Society of London. Series B, Biological Sciences 371 (1708). https://doi.org/10.1098/rstb.2016.0011.

Bateson, Gregory. 1972. Steps to an Ecology of Mind: Collected essays in Anthropology, Psychiatry, Evolution, and Epistemology. University of Chicago Press.

Björkqvist, K., \& Österman, K. (2018). Sex differences in aggression. In The Routledge International Handbook of Human Aggression (pp. 19-30). Routledge.

Bourdieu, Pierre. 1990a. "Structures, Habitus, Practices.” The Logic of Practice, 52-65. 1990b. The Logic of Practice. Stanford University Press.

BrckaLorenz, A., J. Clark, and S. Hurtado. 2017. "Exploring Student Engagement, Gender Identity, and Sexual Orientation,” March. https://scholarworks.iu.edu/dspace/handle/2022/23874.

Bruineberg, Jelle, Julian Kiverstein, and Erik Rietveld. 2018. "The Anticipating Brain Is Not a 2417-44.

Bruineberg, Jelle, and Erik Rietveld. 2014. "Self-Organization, Free Energy Minimization, and

Buss, David M. 1990. "Unmitigated Agency and Unmitigated Communion: An Analysis of the

762 https://doi.org/10.1007/bf00288234.

763 Butler, Judith. 2009. "Performativity, Precarity and Sexual Politics.” AIBR. Revista de Antropología 764 Iberoamericana. https://doi.org/10.11156/aibr.040303e.

765 Cheryan, Sapna. 2012. "Understanding the Paradox in Math-Related Fields: Why Do Some Gender

766 Gaps Remain While Others Do Not?” Sex Roles. https://doi.org/10.1007/s11199-011-0060-z.

767 Choudhury, Suparna, and Jan Slaby. 2011. Critical Neuroscience: A Handbook of the Social and

768 Cultural Contexts of Neuroscience. John Wiley \& Sons.

769 Christopher, Karen. 2013. “African Americans' and Latinas’ Mothering Scripts: An Intersectional

770 Analysis.” Notions of Family: Intersectional Perspectives. https://doi.org/10.1108/s1529-

$771 \quad 2126(2013) 0000017012$.

772 Cisek, Paul. 2019. "Resynthesizing Behavior through Phylogenetic Refinement." Attention,

773 Perception \& Psychophysics 81 (7): 2265-87.

774

775

776

777

778
Code, Jillianne R., and Nicholas E. Zaparyniuk. 2010. "Social Identities, Group Formation, and the Analysis of Online Communities.” Social Computing. https://doi.org/10.4018/978-1-60566-9847.ch086.

Coney, Nicholas Jkmk. 2015. "Performing Genders: A Study of Gender Fluidity." http://digitalcommons.linfield.edu/cgi/viewcontent.cgi?article=1008\&context=soanstud_theses.

This is a provisional file, not the final typeset article 
779 Connell, Raewyn W. 2014. Gender and Power: Society, the Person and Sexual Politics. John Wiley 780 \& Sons.

781 Conrad, Browyn Kara. 2006. "Neo-Institutionalism, Social Movements, and the Cultural

782 Reproduction of A Mentalité: Promise Keepers Reconstruct the Madonna/Whore Complex." The

783 Sociological Quarterly. https://doi.org/10.1111/j.1533-8525.2006.00047.x.

784 Constant, Axel, Maxwell J. D. Ramstead, Samuel P. L. Veissière, and Karl J. Friston. 2019.

785 "Regimes of Expectations: An Active Inference Model of Social Conformity and Human Decision

786 Making.” Frontiers in Psychology 10 (March): 679.

787 Corcoran-Nantes, Yvonne, and Ken Roberts. 1995. "?We've Got One of Those?: The Peripheral

788 Status of Women in Male Dominated Industries." Gender, Work \& Organization.

789 https://doi.org/10.1111/j.1468-0432.1995.tb00024.x.

790 Crenshaw, Kimberlé. 2019. “'Difference’ through Intersectionality 1.” Dalit Feminist Theory.

791 https://doi.org/10.4324/9780429298110-15.

792 Damasio, Antonio. 2018. “The Biological Origins Of Culture.” New Perspectives Quarterly: NPQ 35

793 (2): 22-25.

794 Delmar, Charlotte. 1970. "“Generalizability” as Recognition: Reflections on a Foundational Problem

795 in Qualitative Research.” Qualitative Studies. https://doi.org/10.7146/qs.v1i2.3828.

796 Deutsch, Madeline B. 2016. "Making It Count: Improving Estimates of the Size of Transgender and

797 Gender Nonconforming Populations.” LGBT Health. https://doi.org/10.1089//gbt.2016.0013.

798 Di Paolo, Ezequiel A., Elena Clare Cuffari, and Hanne De Jaegher. 2018. Linguistic Bodies: The

799 Continuity between Life and Language. MIT Press.

800 Dwyer, Rachel E. 2013. “The Care Economy? Gender, Economic Restructuring, and Job Polarization

801 in the U.S. Labor Market.” American Sociological Review 78 (3): 390-416.

802 Edgell, Penny, and Danielle Docka. 2007. "Beyond the Nuclear Family? Familism and Gender

803 Ideology in Diverse Religious Communities." Sociological Forum. https://doi.org/10.1111/j.1573-

804 7861.2006.00003.x.

805 Flintoff, A., H. Fitzgerald, and S. Scraton. 2008. "The Challenges of Intersectionality: Researching

806 Difference in Physical education1." International Studies in Sociology of Education.

807 https://doi.org/10.1080/09620210802351300.

808 Friston, Karl. 2019. "A Free Energy Principle for a Particular Physics.” arXiv [q-bio.NC]. arXiv.

809 http://arxiv.org/abs/1906.10184.

810 Friston, Karl, James Kilner, and Lee Harrison. 2006. "A Free Energy Principle for the Brain."

811 Journal of Physiology, Paris 100 (1-3): 70-87.

812 Gabbard, Glen O., and Sallye Wilkinson. 1996. "Nominal Gender and Gender Fluidity in the

813 Psychoanalytic Situation.” Gender and Psychoanalysis 1 (4): 463-81. 
814 Gardiner, Maria, and Marika Tiggemann. 1999. “Gender Differences in Leadership Style, Job Stress

815 and Mental Health in Male - and Female - Dominated Industries.” Journal of Occupational and

816 Organizational Psychology. https://doi.org/10.1348/096317999166699.

817 Geary, David C. 2010. Male, Female: The Evolution of Human Sex Differences. American

818 Psychological Association.

819 Genesee, Fred. 1982. "The Social Psychological Significance of Code Switching in Cross-Cultural

820 Communication.” Journal of Language and Social Psychology.

821 https://doi.org/10.1177/0261927x8200100102.

822 Gibson, James Jerome. 1979. The Ecological Approach to Visual Perception. Psychology Press.

823 Giddens, Anthony. 1984. The Constitution of Society: Outline of the Theory of Structuration.

824 University of California Press.

825 Goffman, Erving. 1978. "The Presentafion of Self in Everyday Life. 1959.” Repr. Harmondsworth,

826 Middlesex.

827 Griskevicius, Vladas, Noah J. Goldstein, Chad R. Mortensen, Robert B. Cialdini, and Douglas T.

828 Kenrick. 2006. "Going along versus Going Alone: When Fundamental Motives Facilitate Strategic

829 (non)conformity.” PsycEXTRA Dataset. https://doi.org/10.1037/e633962013-016.

830 Hazelkorn, Ellen. 2018. "Gender and Higher Education: Increasing Exposure of Harassment and Pay

831 Gaps.” International Higher Education. https://doi.org/10.6017/ihe.2018.94.10515.

832 Herron, Mary Jane, William G. Herron, and Candace L. Schultz. 1983. "Sexual

833 Dominance/Submission, Gender and Sex-Role Identification." Perceptual and Motor Skills.

834 https://doi.org/10.2466/pms.1983.56.3.931.

835 Hornsey, Matthew J., Louise Majkut, Deborah J. Terry, and Blake M. McKimmie. 2003. “On Being 836 Loud and Proud: Non-Conformity and Counter-Conformity to Group Norms." The British Journal of 837 Social Psychology / the British Psychological Society 42 (Pt 3): 319-35.

838 Istenič, Majda Černič. 2007. “Attitudes Towards Gender Roles and Gender Role Behaviour Among 839 Urban, Rural, and Farm Populations in Slovenia.” Journal of Comparative Family Studies 38 (3):

$840 \quad 477-96$.

841 Jain, Priya, Gyanesh Kumar Tiwari, Ishdutta Awasthi, and A. Chaubey. 2018. "Menstrual Distress

842 and Attitude towards Femininity of Rural and Urban Adolescent Girls." Madhya Bharti 74: 222-33.

843 Johnson, Mark. 2007. “"The Stone That Was Cast out Shall Become the Cornerstone': The Bodily

844 Aesthetics of Human Meaning." Journal of Visual Art Practice 6 (2): 89-103.

845 Jordan-Young, Rebecca M., and Katrina Karkazis. 2019. Testosterone: An Unauthorized Biography. 846 Harvard University Press.

847 Kaplan, Raphael, and Karl J. Friston. 2018. "Planning and Navigation as Active Inference."

848 Biological Cybernetics 112 (4): 323-43. 
849 Kim, Min Jung, Sang Do Shin, William Marvin McClellan, Bryan McNally, Young Sun Ro, Kyoung

850 Jun Song, Eui Jung Lee, et al. 2014. "Neurological Prognostication by Gender in out-of-Hospital

851 Cardiac Arrest Patients Receiving Hypothermia Treatment." Resuscitation 85 (12): 1732-38.

852 Kirchhoff, Michael D., and Julian Kiverstein. 2019. “The Extended Mind.” Extended Consciousness

853 and Predictive Processing. https://doi.org/10.4324/9781315150420-2.

854 Krane, Vikki. 2018. Sex, Gender, and Sexuality in Sport: Queer Inquiries. Routledge.

855 Laner, Mary Riege, and Nicole A. Ventrone. 2000. "Dating Scripts Revisited." Journal of Family

856 Issues. https://doi.org/10.1177/019251300021004004.

857 Lawson, Victoria. 1995. "The Politics of Difference: Examining the Quantitative/Qualitative

858 Dualism in Post-Structuralist Feminist Research*." The Professional Geographer.

859 https://doi.org/10.1111/j.0033-0124.1995.00449.x.

860 Linstead, Alison, and Joanna Brewis. 2004. "Editorial: Beyond Boundaries: Towards Fluidity in

861 Theorizing and Practice." Gender, Work and Organization. https://doi.org/10.1111/j.1468-

862 0432.2004.00237.x.

863 Mangan, Katherine. 2012. "Despite Efforts to Close Gender Gaps, Some Disciplines Remain

864 Lopsided." The Chronicle of Higher Education, October. http://chronicle.com/.

865 Maturana, H. R., and F. J. Varela. 1991. Autopoiesis and Cognition: The Realization of the Living.

866 Springer Science \& Business Media.

867 McCarthy, Karen, Meghan Ballog, Maria Mayela Carranza, and Katie Lee. 2020. "Doing Nonbinary

868 Gender: The Occupational Experience of Nonbinary Persons in the Environment." Journal of

869 Occupational Science. https://doi.org/10.1080/14427591.2020.1804439.

870 Mcfarland, Daniel, and Heili Pals. 2005. "Motives and Contexts of Identity Change: A Case for

871 Network Effects.” Social Psychology Quarterly. https://doi.org/10.1177/019027250506800401.

872 Moreno, Eva M., Kara D. Federmeier, and Marta Kutas. 2002. "Switching Languages, Switching

873 Palabras (words): An Electrophysiological Study of Code Switching.” Brain and Language 80 (2):

874 188-207.

875 Murphy, Amy S., Melanie A. Acosta, and Brianna L. Kennedy-Lewis. 2013. “'I'm Not Running

876 around with My Pants Sagging, so How Am I Not Acting like a Lady?': Intersections of Race and

877 Gender in the Experiences of Female Middle School Troublemakers." The Urban Review.

878 https://doi.org/10.1007/s11256-013-0236-7.

879 Nicholls, Walter J. 2008. "The Urban Question Revisited: The Importance of Cities for Social

880 Movements.” International Journal of Urban and Regional Research 32 (4): 841-59.

881 Österman, K., and K. Björkqvist. 2018. "Sex Differences in Aggression.” The Routledge

882 International Handbook of Human Aggression, 47-58. 
883 Paechter, Carrie. 2003. "Masculinities and Femininities as Communities of Practice." Women's

884 Studies International Forum 26 (1): 69-77.

885 . 2006. "Power, Knowledge and Embodiment in Communities of Sex/gender Practice."

886 Women's Studies International Forum 29 (1): 13-26.

887 Pitts-Taylor, Victoria. 2013. "I Feel Your Pain: Embodied Knowledges and Situated Neurons."

888 Hypatia. https://doi.org/10.1111/hypa.12049.

889 Pompper, Donnalyn. 2016. Rhetoric of Femininity: Female Body Image, Media, and Gender Role

890 Stress/Conflict. Lexington Books.

891 Powell, Crystal. 2018. "Bias, Employment Discrimination, and Black Women's Hair: Another Way

892 Forward.” BYU L. Rev., 933.

893 Ramstead, Maxwell J. D., Samuel P. L. Veissière, and Laurence J. Kirmayer. 2016. “Cultural

894 Affordances: Scaffolding Local Worlds Through Shared Intentionality and Regimes of Attention."

895 Frontiers in Psychology 7 (July): 1090.

896 Randall, William L., and Cassandra Phoenix. 2009. "The Problem with Truth in Qualitative

897 Interviews: Reflections from a Narrative Perspective.” Qualitative Research in Sport and Exercise.

898 https://doi.org/10.1080/19398440902908993.

899 Reinharz, Shulamit, Mary Margaret Fonow, and Judith A. Cook. 1992. "Beyond Methodology:

900 Feminist Scholarship as Lived Research.” Contemporary Sociology.

901 https://doi.org/10.2307/2075514.

902 Richards, Christina, Walter Pierre Bouman, Leighton Seal, Meg John Barker, Timo O. Nieder, and

903 Guy T'Sjoen. 2016. “Non-Binary or Genderqueer Genders.” International Review of Psychiatry 28

904 (1): 95-102.

905 Richardson, Diane. 2010. "Youth Masculinities: Compelling Male Heterosexuality." The British

906 Journal of Sociology. https://doi.org/10.1111/j.1468-4446.2010.01339.x.

907 Satinsky, Emily, and Denise Nicole Green. 2016. "Negotiating Identities in the Furry Fandom

908 through Costuming." Critical Studies in Men???s Fashion. https://doi.org/10.1386/csmf.3.2.107_1.

909 Scaptura, Maria N., and Kaitlin M. Boyle. 2020. "Masculinity Threat, 'Incel' Traits, and Violent

910 Fantasies Among Heterosexual Men in the United States.” Feminist Criminology.

911 https://doi.org/10.1177/1557085119896415.

912 Schank, Roger C., and Robert P. Abelson. 2013. Scripts, Plans, Goals, and Understanding: An

913 Inquiry Into Human Knowledge Structures. Psychology Press.

914 Schippers, Mimi. 2007. "Recovering the Feminine Other: Masculinity, Femininity, and Gender

915 Hegemony." Theory and Society 36 (1): 85-102.

916 Sewell, William H. 1992. “A Theory of Structure: Duality, Agency, and Transformation.” The

917 American Journal of Sociology 98 (1): 1-29. 
918

919

920

921

922

923

924

925

926

927

928

929

930

931

932

933

934

935

936

937

938

939

940

941

942

943

944

945

946

947

948

949

950

—. 1998. "Culture, Structure, Agency, and Transformation." The New American Cultural Sociology. https://doi.org/10.1017/cbo9780511520808.017.

Shields, Stephanie A. 2008. “Gender: An Intersectionality Perspective.” Sex Roles. https://doi.org/10.1007/s11199-008-9501-8.

Shome, Raka. 2011. “Global Motherhood': The Transnational Intimacies of White Femininity.” Critical Studies in Media Communication. https://doi.org/10.1080/15295036.2011.589861.

Simon, William, and John H. Gagnon. 1986. "Sexual Scripts: Permanence and Change." Archives of Sexual Behavior. https://doi.org/10.1007/bf01542219.

Staples, J. M. 2005. "What's Love Got to Do with It? Reading 'Black Femininity' in a Media Text." Perspectives on Urban Education 3 (2): 1-12.

Sterling, Peter. 2012. “Allostasis: A Model of Predictive Regulation.” Physiology \& Behavior 106 (1): 5-15.

Sullivan, Jas M., and Ashraf Esmail. 2012. African American Identity: Racial and Cultural Dimensions of the Black Experience. Lexington Books.

Thompson, Evan. 2007. Mind in Life. Harvard University Press.

Thompson, Vetta L. Sanders. 2001. "The Complexity of African American Racial Identification." Journal of Black Studies 32 (2): 155-65.

Turing, Alan Mathison. 1936. "On Computable Numbers, with an Application to the Entscheidungsproblem.” Journal of Math-for-Industry 58 (345-363): 5.

Valentine, Gill. 2007. "Theorizing and Researching Intersectionality: A Challenge for Feminist Geography*." The Professional Geographer. https://doi.org/10.1111/j.1467-9272.2007.00587.x.

Valocchi, Stephen. 2005. "Not Yet Queer Enough: The Lessons of Queer Theory for the Sociology of Gender and Sexuality." Gender \& Society: Official Publication of Sociologists for Women in Society 19 (6): 750-70.

Varela, Francisco J., Evan Thompson, and Eleanor Rosch. 2016. The Embodied Mind: Cognitive Science and Human Experience. MIT Press.

von Uexküll, Jakob. 1957. A Stroll Through the Worlds of Animals and Men: A Picture Book of Invisible Worlds. In Instinctive Behavior: The Development of a Modern Concept, edited and translated by Claire H. Schiller, New York: International Universities Press.

Wainwright, David. 1997. "Can Sociological Research Be Qualitative, Critical and Valid?" Qualitative Report 3 (2). http://kar.kent.ac.uk/24709/.

Weichold, Martin, and Gerhard Thonhauser. 2020. "Collective Affordances.” Ecological Psychology. https://doi.org/10.1080/10407413.2019.1695211. 
951 Wright, Tessa. 2016. "Women's Experience of Workplace Interactions in Male-Dominated Work:

952 The Intersections of Gender, Sexuality and Occupational Group.” Gender, Work \& Organization.

953 https://doi.org/10.1111/gwao.12074. 